\title{
Top-Down Activation of Spatiotopic Sensory Codes in Perceptual and Working Memory Search
}

\author{
Bo-Cheng Kuo ${ }^{1}$, Anna Christina Nobre ${ }^{2}$, Gaia Scerif ${ }^{2}$, and Duncan E. Astle ${ }^{3}$
}

\begin{abstract}
A critical requirement of an efficient cognitive system is the selection and prioritization of relevant information. This occurs when selecting specific items from our sensory inputs, which then receive preferential status at subsequent levels of processing. Many everyday tasks also require us to select internal representations, such as a relevant item from memory. We show that both of these types of search are underpinned by the spatiotopic activation of sensory codes, using both fMRI and MEG data. When individuals searched for perceived and remembered targets, the MEG data highlighted a sensor level electrophysiological effect that reflects the contralateral organization of the visual system - namely, the N2pc. The fMRI data were used to identify a network of frontoparietal areas common to both types of search,
\end{abstract}

\section{INTRODUCTION}

Efficient goal-directed behavior depends upon our ability to identify relevant information in our external environment. "Visual search" is a paradigm commonly used for exploring the cognitive and neural processes by which we do this (Treisman \& Gelade, 1980). Items are visually presented, and participants must search for the presence of a specific target item, which is only present on a subset of trials. This paradigm has been intensively studied, with researchers varying numerous parameters, such as increasing the number of nontarget items (Treisman \& Sato, 1990) or their distinctiveness (Duncan \& Humphreys, 1989). The result is an extensive literature in which the mechanisms that afford visual search are keenly debated (Cunningham \& Wolfe, 2014; Eimer, 2014; Wolfe, Võ, Evans, \& Greene, 2011; Chelazzi, 1999; Wolfe, 1994; Duncan \& Humphreys, 1989; Wolfe, Cave, \& Franzel, 1989; Treisman \& Gelade, 1980).

The role of spatial attention varies across the various theoretical accounts of visual search, from a restricted role in target selection all the way to a critical role in directing the search itself (Chelazzi, 1999). ERP recordings provide a key piece of evidence for the role of spatially specific attention at some stage of the search process. Between 200 and

\footnotetext{
${ }^{1}$ National Taiwan University, ${ }^{2}$ University of Oxford, ${ }^{3} \mathrm{MRC}$ Cognition and Brain Sciences Unit, Cambridge, UK

as well as the early visual areas activated by the search display. We then combined fMRI and MEG data to explore the temporal dynamics of functional connections between the frontoparietal network and the early visual areas. Searching for a target item resulted in significantly enhanced phase-phase coupling between the frontoparietal network and the visual areas contralateral to the perceived or remembered location of that target. This enhancement of spatially specific phase-phase coupling occurred before the N2pc effect and was significantly associated with it on a trialby-trial basis. The combination of these two imaging modalities suggests that perceptual and working memory search are underpinned by the synchronization of a frontoparietal network and the relevant sensory cortices.
400 msec after the onset of the search array, potentials are more negative over electrode sites contralateral, relative to ipsilateral, to the target location. This well-replicated effect is called the N2pc (Eimer \& Grubert, 2014; Tollner, Gramann, Muller, Kiss, \& Eimer, 2008; Woodman, Kang, Rossi, \& Schall, 2007; Luck \& Hillyard, 1994). The specific aspect of visual search indexed by the N2pc is still debated, with some suggesting that it reflects the spatial orienting of attention (Luck \& Hillyard, 1994) and others that it reflects spatial selection of targets based on template matching (Eimer \& Grubert, 2014; Brignani, Lepsien, \& Nobre, 2010; Kuo, Rao, Lepsien, \& Nobre, 2009; Eimer, 1996). Despite disagreements about details, because the organization of the N2pc reflects the contralateral organization of the human visual system, its presence provides strong evidence for the spatially specific activation of sensory codes during visual search. Importantly, a number of recent studies have shown that an N2pc is also elicited when participants search for a target within working memory (WM; Dell'Acqua, Sessa, Toffanin, Luria, \& Jolicoeur, 2010; Eimer \& Kiss, 2010; Astle, Scerif, Kuo, \& Nobre, 2009; Kuo et al., 2009). This implies that both visual and mnemonic search involve some comparable modulation that reflects the spatial organization of the original sensory input. However, we know very little about how these sensory codes are activated while searching for a visual or remembered target or how this process is reflected by the sensor level N2pc effect. We aim to address this in the current study. 
A popular neurally inspired account for visual search suggests that it depends on a parallel, competitive mechanism (Duncan \& Humphreys, 1989). The neural representations of visual items compete, and an item is selected as a target when its representation wins that competitive process. This process can be guided by a top-down biasing mechanism: A remembered template of the target is used to bias the receptive fields associated with that target, so any display items that also activate those receptive fields will receive a competitive advantage. This bias is applied in a graded fashion, with the strength of bias that items receive being proportional to their representational overlap with the template. If many display items have highly similar neural representations to the target or if no single receptive field is uniquely associated with the target, then this competitive process will take longer and the degree of bias required will be greater (Desimone \& Duncan, 1995). Multimodal brain regions, centering on areas of frontal and parietal cortex, are thought to be responsible for generating these top-down biasing signals (Becker, Grubert, \& Dux, 2014; Preston, Guo, Das, Giesbrecht, \& Eckstein, 2013; Weidner, Krummenacher, Reimann, Muller, \& Fink, 2009; Buschman \& Miller, 2007; Nobre, Coull, Walsh, \& Frith, 2003; Miller \& Cohen, 2001; Kanwisher \& Wojciulik, 2000; Chelazzi, Duncan, Miller, \& Desimone, 1998; Corbetta, Shulman, Miezin, \& Petersen, 1995; Chelazzi, Miller, Duncan, \& Desimone, 1993). To bias ongoing representational competition, this network provides input into the sensory areas carrying the target-related content. Neuronal synchrony has been proposed to provide a possible means by which this competitive biasing might occur (Siegel, Donner, Oostenveld, Fries, \& Engel, 2008; Womelsdorf et al., 2007; Fries, Reynolds, Rorie, \& Desimone, 2001): By selectively synchronizing rhythmic activity in relevant sensory cortex with that of subsequent visual areas, frontoparietal cortex can allow relevant representations to pass further along the ventral visual processing stream. By contrast, representations within areas of sensory cortex that are oscillating asynchronously will not proceed for further processing. This spatially and temporally specific pattern of neuronal coupling allows frontoparietal cortex to gate the flow of sensory information according to its relevance (Fries, 2005, 2009, 2015; Marshall, Bergmann, \& Jensen, 2015; Klimesch, 2012). The purpose of this study was to test whether selective phase coupling between task control areas and relevant ventral visual areas can provide a neurophysiological mechanism underlying spatial biases in visual and mnemonic search in the human brain.

First, we established the neural architectures involved in visual and WM search in an fMRI experiment, identifying the frontoparietal and early visual cortical areas involved. Second, we characterized the spatially specific modulation related to target selection using sensor level recordings in an MEG experiment-equivalent to the N2pc. We subsequently combined the fMRI and MEG data: We used source-projected MEG data to explore the coordination of dynamic neural activity between the frontoparietal and visual areas identified by fMRI. Specifically, we explored whether the frontoparietal network selectively coupled with early visual areas according to the spatial location of the target within the search display. We tested the hypothesis that there would be stronger phase coupling between frontoparietal areas and the visual areas contralateral, relative to ipsilateral to the target. Finally, we explored the trialto-trial relationship between this phase coupling in source space and the sensor level N2pc effect.

\section{METHODS}

The same basic task design was used across both fMRI and MEG experiments. The two studies recruited separate participants and were conducted in Taiwan and the UK, respectively. In each experiment, participants completed visual search and WM search conditions with arrays of two or four items (Astle et al., 2009; Kuo et al., 2009). On visual search trials, participants were first presented with a single item at fixation. After a brief delay, they searched covertly for this single item in an upcoming array of either two or four items. On WM search trials, participants first viewed an array of two or four items, which had to be maintained in WM. After a short delay, a single item appeared at fixation, and participants indicated whether this item had been present in the preceding array or not, requiring them to search retrospectively their memory of the array presented earlier that trial. Search type (visual, WM), array size (two-item, four-item), and the correct search response (target-present, target-absent) were manipulated in a factorial design. Both search types and array sizes were equally probable, and the order of the trial types was randomly intermixed within experimental blocks. A task schematic is shown in Figure 1.

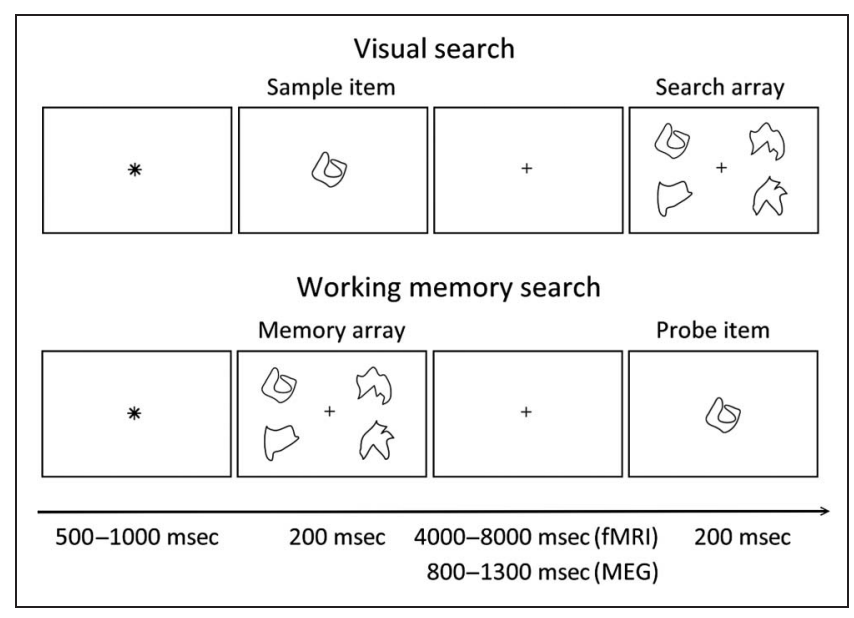

Figure 1. Schematic of experimental trials: visual search (top) and WM search (bottom) used in fMRI and MEG experiments. In both types of search, the delay between presentation of the first and second displays varied between 4000 and $8000 \mathrm{msec}$ for the fMRI experiment and between 800 and $1300 \mathrm{msec}$ for the MEG experiment 


\section{fMRI Experiment}

\section{Participants}

Eighteen healthy, right-handed (Oldfield, 1971) volunteers with normal or corrected-to-normal visual acuity participated in the fMRI experiment. They all provided written informed consent before the study and were reimbursed for their time. Data from two participants were excluded due to excessive head movement in the scanner (greater than $5 \mathrm{~mm}$ ). The behavioral and fMRI analyses were performed on the remaining 16 participants (age range $=20-27$ years, 10 women), who all showed above-chance behavioral performance across all task conditions ( $>55 \%$ correct trials). All experimental methods and procedures had ethical approval from the research ethics office of National Taiwan University.

\section{Stimuli}

Search arrays were composed of abstract shape stimuli. Eight stimuli were selected from a set of 100 novel and difficult to verbalize closed shape contours previously developed by Endo, Saiki, Nakao, and Saito (2003). Each shape was $1.72^{\circ}$ visual angle in size and when presented as part of an array was positioned randomly in one of the four possible peripheral locations of an invisible $2 \times$ 2 matrix. Stimulus locations were centered at $2.58^{\circ}$ eccentricity and $2^{\circ}$ elevation. Two-item arrays contained stimuli presented along a diagonal (upper left and lower right, or upper right and lower left). All stimulus contours were white. A black background was used throughout the experiment.

\section{Task Design}

Each trial began with the onset of a centrally displayed asterisk (500-1000 msec duration), which signaled the onset of the trial. On visual search trials, one shape item was initially presented at the center of the screen (for $200 \mathrm{msec}$ ). After a randomized variable interval (40008000 msec duration), it was followed by an array of two or four peripherally located shapes (200 msec). Participants searched covertly for the initial shape within the array and indicated whether the shape was present or absent. On WM search trials, an array of two or four peripheral shapes was initially presented (for $200 \mathrm{msec}$ ) and, after a randomized variable interval (4000-8000 msec), was followed by a single centrally presented shape item (for $200 \mathrm{msec}$ ). The interval between trials, which included a 1000-msec response period, was varied randomly between 4500 and 8500 msec. These long and jittered interstimulus and intertrial intervals enabled disambiguation of the fMRI responses.

To identify ROIs in early visual cortex, participants also performed a visual localizer task (Figure 2A). Participants were instructed to view the stimuli passively without making any responses. In the visual localizer task, the
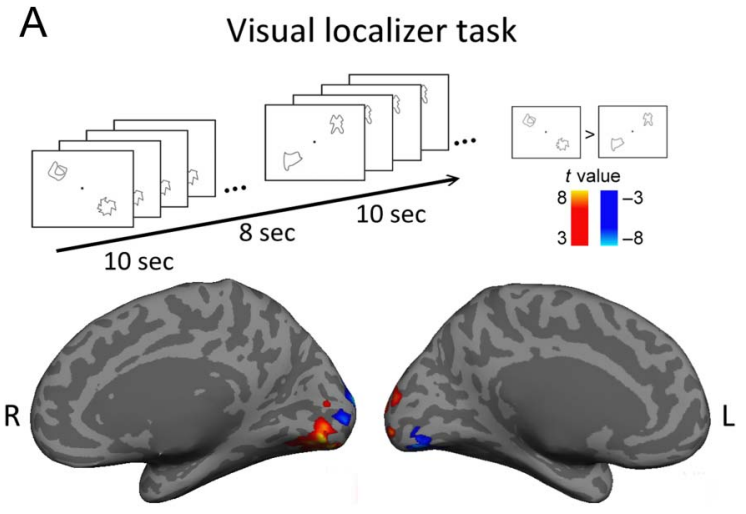

B Common activations for visual search and working memory search
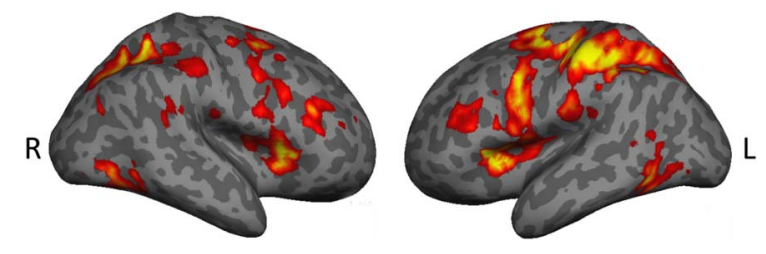

Figure 2. Results for fMRI experiment. (A) In the visual localizer task, we showed two-item arrays containing the same stimulus items presented along a diagonal (upper left and lower right, or upper right and lower left) at the same locations of the main search tasks. The activation corresponding to the stimulus locations was found in early visual areas (red color scale: upper left and lower right $>$ upper right and lower left; blue color scale: upper right and lower left $>$ upper left and lower right). (B) We showed common activations of the dorsal frontoparietal network for both visual and WM search. Activations were also observed in occipito-temporal areas. $\mathrm{L}=$ left hemisphere; $\mathrm{R}=$ right hemisphere. The FreeSurfer software package (surfer.nmr.mgh. harvard.edu) was used for projecting results onto flattened cortical surfaces.

same shape stimuli used in the search tasks were presented in two-item arrays, arranged along a diagonal (left upper and right lower, or left lower and right upper) on a black background in a blocked design. Eccentricity and elevation were the same as in the search tasks. Each two-item array was presented for $500 \mathrm{msec}$ followed by a 500-msec blank interval, and each array was repeated 10 times in each block (10-sec duration for each block; Kuo, Stokes, Murray, \& Nobre, 2014). The same shape stimuli and locations were used in each block. The interval between blocks was $8000 \mathrm{msec}$. The purpose of this localizer task was to isolate the areas of early visual cortex that respond selectively to the visual presentation of display items at these specific locations. These visual areas were subsequently used in our combined fMRI/ MEG analysis.

\section{Experimental Procedure}

Before fMRI scanning, each participant was given written as well as verbal instructions about the task requirements 
and completed one practice session (32 trials, 16 trials for each search task) outside the scanner to ensure that they could perform the task as instructed. Participants were also instructed to maintain fixation on a small marker at the center of the screen during the active parts of the experiment and to respond as accurately as possible. All participants responded using their right hand. Participants were also asked to minimize eye and body movement throughout the experiment. They completed four runs of the task (48 trials per run), completing 192 trials in total (48 target-present and 48 target-absent trials in each search type) and two runs of the visual localizer task (24 blocks per run, 48 blocks in total). Scanning time for each participant was approximately $1 \mathrm{hr}$ and $30 \mathrm{~min}$.

Stimuli were presented with Presentation software (Neurobehavioral Systems, Albany, NY). Visual stimuli were rear-projected on to a screen via a Hitachi LCD projector at $1024 \times 768$ resolution and a $60-\mathrm{Hz}$ refresh rate. Participants in the MR scanner viewed the screen through a mirror fixed to the head coil. If required, MR-compatible corrective lenses were used to ensure normal visual acuity during the experiment. Behavioral responses were recorded using an MR-compatible Current Designs response button box.

\section{fMRI Acquisition and Scanning Parameters}

Images were acquired on a Siemens 3-T MAGNETOM Skyra MRI scanner (Berlin, Germany) with a 20-channel head coil at the National Chengchi University, Taipei, Taiwan. Functional data were collected using a T2*weighted gradient EPI sequence (repetition time $=$ $2000 \mathrm{msec}$, echo time $=35 \mathrm{msec}$, flip angle $=90^{\circ}$, field of view $[\mathrm{FOV}]=192 \times 192 \mathrm{~mm}$, matrix size $=64 \times 64)$ to measure BOLD response. EPI images were obtained from 32 contiguous axial-oblique slices $(3 \mathrm{~mm}$ thick with no gap) acquired in descending order in-plane resolution of $3 \times 3 \mathrm{~mm}$. High-resolution anatomical images $(1 \times 1 \times$ $1 \mathrm{~mm}$ ) were also acquired for each participant using a T1weighted MP-RAGE sequence $(\mathrm{FOV}=192 \times 192 \mathrm{~mm})$.

\section{fMRI Analysis}

The fMRI data preprocessing and subsequent analyses were carried out with SPM5 software (Wellcome Trust Centre for Neuroimaging, University College London, London, UK) in MATLAB (The MathWorks, Natick, MA). The first four volumes of each run were discarded to allow for magnetic saturation effects. The remaining functional images were corrected for head movement artifact and timing differences in slice acquisitions. Preprocessed functional images were coregistered to the individual anatomical image and normalized to the standard SPM/ Montreal Neurological Institute (MNI) brain template (Collins, Neelin, Peters, \& Evans, 1994) and resampled to a 2-mm isotropic voxel size. Normalized images were spatially smoothed with a Gaussian kernel of 8-mm
FWHM to accommodate any anatomical variability across participants (Hopfinger, Buchel, Holmes, \& Friston, 2000; Worsley et al., 1996). The time series data were then highpass filtered with a frequency cutoff at $128 \mathrm{sec}$ and prewhitened by means of an autoregressive model AR(1).

For the whole-brain analysis, statistical inference was based on a random-effects approach. At the individual level, the data of each participant were analyzed using the general linear model (GLM) by fitting the time series data with the canonical hemodynamic response function modeled at the events of interest. The model included all trial events and variables: probe item and search array, separated according to the number of items in the array, target presence or absence, and error trials. Movement parameters were also used to construct six regressors to model variance caused by head movements during scanning. In our comparisons, we focused our analyses on the events during which participants searched for the target either in the visual array or in the memory representation of that array-that is, array-locked for the visual search trials and probe-locked for the WM search trials. Linear contrasts were calculated to characterize responses of interest for single participants' data at the first level. Group results were subsequently determined using a random-effects analysis at the second level. To determine visual and WM search-related effects, we contrasted two search events based on the target-present trials with the implicit baseline, respectively (e.g., visual search vs. implicit baseline; WM search vs. implicit baseline). A conjunction analysis was then conducted (Nichols, Brett, Andersson, Wager, \& Poline, 2005) to test for any areas of common activation during visual and WM search.

We also analyzed the fMRI data from the visual localizer task using a GLM. The purpose of the localizer task was to identify the early visual areas that were selectively responsive to the locations of the visual stimuli in the search tasks. We selected the early visual areas that were sensitive to the four visual field locations by contrasting activations in the two blocks (upper left and lower right vs. lower left and upper right). These functional localizers were then used as ROIs to guide our subsequent MEG analysis.

The estimations for each contrast from the search and localizer tasks were then entered into a standard SPM group level analysis in which the participants were treated as a random variable using a one-sample $t$ test. We used a threshold of $p<.05$, correcting for multiple comparisons using the false discovery rate.

\section{MEG Experiment}

\section{Participants}

Fourteen healthy volunteers with normal or corrected-tonormal visual acuity participated in this experiment. All participants were right-handed, according to the Edinburgh handedness inventory (Oldfield, 1971). Written informed 
consent was obtained from all participants before the study, and they were financially reimbursed for their time. The behavioral and MEG data were only analyzed from 11 participants (five women and six men, age range = 20-34 years), who all showed above-chance behavioral performance ( $>55 \%$ correct trials) and had a sufficient number of artifact-free MEG trials across all task conditions (>30 trials). The other three participants did not meet these criteria. All experimental methods and procedures were approved by the central university research ethics committee of the University of Oxford.

\section{Task Design}

The experimental design and the stimuli used in the MEG experiment were identical to those in the fMRI experiment, but with short interstimulus and intertrial intervals, as in a previous study (Kuo et al., 2009). The interval between probe and search arrays varied randomly between 800 and $1300 \mathrm{msec}$. The interval between trials, which included a 1000-msec response period, varied randomly between 2500 and $3500 \mathrm{msec}$. There were 768 trials in total (96 target-present and 96 target-absent trials in each search type and array size).

\section{Experimental Procedure}

Participants were comfortably seated in a magnetically and electrically shielded room, $100 \mathrm{~cm}$ from the screen on which the stimuli were projected. Each participant was given written as well as verbal instructions about the task requirements and completed one additional practice block with 16 practice trials for each search task before the actual experiment. They were also instructed to maintain fixation on a small marker and to respond as accurately and quickly as possible. All participants responded using their right hand. Participants completed four runs (including 12 miniblocks of 16 trials for each run), with the rest periods being self-paced. MEG recording time for each participant was approximately $1 \mathrm{hr}$ and $10 \mathrm{~min}$.

\section{MEG Acquisition and Recording Parameters}

MEG data were acquired on a 306-channel VectorView system (Elekta Neuromag, Stockholm, Sweden), with 102 magnetometer and 204 orthogonal planar gradiometers (pairs of sensors measuring the longitudinal and latitudinal derivatives of the magnetic field) at the Oxford Centre for Human Brain Activity. The data were sampled at $1000 \mathrm{~Hz}$ and band-pass filtered at $0.03-330 \mathrm{~Hz}$. Head position was monitored at the start and at the end of each of the four experimental runs using four head position indicator coils placed on the scalp, used as indicators of head position in the MEG helmet. We also used a digitizer (Polhemus, Colchester, VT) to digitize three fiducial landmarks (the nasion, left and right preauricular points), four head position indicator coils, and a number of additional head points for further alignment with individual T1-weighted structural MRI or standard MNI template. Vertical eye movements were recorded by MEG-compatible electrodes placed on the supraorbital and infraorbital ridges of the left eye [vertical electrooculogram (VEOG)] and horizontal eye movements by electrodes placed on the outer canthi of the right and left eyes [horizontal electrooculogram (HEOG)]. VEOG and HEOG were recorded and subsequently used to remove trials contaminated by blinks or eye movements.

\section{Structural MRI Acquisition and Scanning Parameters for MEG Analyses}

High-resolution anatomical images $(1 \times 1 \times 1 \mathrm{~mm})$ were available for nine participants using a T1-weighted MPRAGE sequence $(F O V=192 \times 192 \mathrm{~mm})$ on a Siemens 3-T Tim Trio scanner (Berlin, Germany) with a 12-channel head coil at the University of Oxford Centre for Clinical Magnetic Resonance Research. For the remaining participants, we used a standard MNI template.

\section{MEG Data Analysis}

The first MEG preprocessing step involved removing external noise from the data using the signal space separation method implemented in Elekta's Maxfilter software (Albuquerque, New Mexico). MEG analyses were performed using SPM8 software (Wellcome Trust Centre for Neuroimaging, University College London, London, UK), Fieldtrip toolbox (Oostenveld, Fries, Maris, \& Schoffelen, 2011), and the in-house Oxford Centre for Human Brain Activity Software Library (OSL) in MATLAB. The continuous data were down-sampled to $250 \mathrm{~Hz}$ and segmented into epochs from -500 to $1000 \mathrm{msec}$ relative to the onset of the search array for visual search and the onset of the probe item for WM search. In each case, the prestimulus period was used as a baseline. Trials with incorrect behavioral responses were discarded. The epoched data were then visually inspected. The epochs contaminated by eye blinks and movements detected from the VEOG and HEOG were excluded from all analyses. Outlier trials with abnormally high variance in the time domain data were also removed using Fieldtrip's visual artifact rejection tool.

The MEG data analyses comprised two sections: (1) an analysis of sensor level event-related field (ERF), testing for the N2pc, according to target locations in either the visually presented (search arrays for visual search) or remembered array (probe item for WM search), and (2) an fMRI-guided MEG source level phase-coupling analysis, testing for spatially specific functional coupling between frontoparietal network and posterior visual areas.

\section{MEG Sensor Level ERF Analysis}

First, we conducted a sensor space ERF analysis to test for the N2pc-like effects from MEG signals as previous 
studies (Kuo et al., 2009). The artifact-free ERF epochs were low-pass filtered $(40 \mathrm{~Hz})$ and normalized to the baseline period (200 msec) before stimulus presentation. We averaged the ERF data based on target-present trials across the two search types and two target sides (leftward and rightward visual search and WM search) for each participant. ERFs were derived from the onset of the search arrays in the visual search condition; on the other hand, ERFs were derived from the onset of the centrally presented probe item in the WM search condition. ERFs from trials containing perceptual or remembered targets located on the right and left side were then combined by an averaging procedure that preserved the location of MEG sensors relative to the target side (ipsilateral or contralateral). This procedure thus resulted in four conditions-ipsilateral and contralateral to target for visual search and WM search.

Sensor space statistical analyses were performed on the planar gradiometers, 102 latitudinal channels and 102 longitudinal channels, respectively, which are most sensitive to lateralized effects (Mitchell \& Cusack, 2011). ERF differences between sensors ipsilateral versus contralateral to target were tested for every time point during the possible time window of the N2pc between 200 and 400 msec using dependent sample $t$ tests over all sensors. We then corrected for multiple comparisons using a cluster-based nonparametric permutation approach (Maris \& Oostenveld, 2007). We calculated the size of any significant clusters with consecutive $t$ tests that were significant ( $p<.05$, two-tailed) either across neighboring sensors, time points, or both. Finally, we calculated Monte Carlo $p$ values on 1000 random partitions in which ipsilateral and contralateral to target were shuffled for each search type, thereby deriving a null distribution of the cluster sizes that would be achieved by chance. The cluster was treated as significant if its size was significantly unlikely to have occurred by chance $(p<.05$, two-tailed $)$.

\section{fMRI-MEG Source Level Phase-coupling Analysis}

Having identified the presence of a common lateral frontoparietal network for both search types in our fMRI conjunction analysis and localized early visual areas with our fMRI localizer task, we used MEG to test for spatially specific coupling between these areas. First, we coregistered each individual's MEG data based on their T1-weighted structural image or a standard MNI template using digitized scalp locations and fiducials via an iterative closest point algorithm using SPM software. We used a 6-mm grid, constructed within the boundaries of the fMRI results, and then combined the dipoles to produce a single signal for the frontoparietal network and the left and right visual ROIs. We then used a beamformer to extract a broad band signal $(3-100 \mathrm{~Hz})$ from the lateral frontoparietal network identified by the fMRI analysis and visual ROIs. The beamformer combined information from both the magnetometers and planar gradiometers while taking into account the reduced dimensionality of the data introduced by the signal space separation algorithm (Woolrich, Hunt, Groves, \& Barnes, 2011). Beamforming constructs a spatial filter, which is applied to the sensor data to reconstruct the signal at each grid point, with the aim of achieving unit bandpass response at the grid point while minimizing the variance passed from all other locations. In short, the data at the source location of interest are given by multiplying the beamformer weights vector by the original sensor data. The process can be repeated across all grid locations to achieve a complete source reconstruction for all of the dipoles within our network and visual areas. The data covariance matrix was also regularized according to Creg $=\mathrm{C}+\mu \mathrm{I}$, where $\mu$ equals 4 times the minimum eigenvalue of the unregularized data covariance matrix, C. Thus data bandpass-filtered to the frequencies of interest were projected onto source space for each participant.

The beamformer-extracted broadband signal was then decomposed into its constituent time-frequency components using a Morlet Wavelet Transform from 3 to $30 \mathrm{~Hz}$ in $0.5-\mathrm{Hz}$ steps for all trials. We focused on the lower frequencies based on previous findings that demonstrate significant functional coupling in MEG using lower frequencies (Astle, Luckhoo, et al., 2015) and increased discrimination between spurious and genuine connectivity (Luckhoo et al., 2012).

We calculated phase coupling between the frontoparietal network and each of the lateral visual ROIs using circular-circular correlations for each frequency (Pauen \& Ivanova, 2013). Phase-phase coupling provides one means by which distinct cortical areas can be selectively coupled and has been shown to be a mechanism by which attention can gate the flow of relevant information through visual areas (Fries, 2005, 2015; Womelsdorf \& Fries, 2007). We used a 400-msec time window and calculated the phase-phase coupling within the window, moving the window along in 4-msec steps to construct the time course of coupling from 0 to $400 \mathrm{msec}$. Having done this separately across left and right early visual areas, for visual and WM search conditions, we then performed a GLM. This tested for any significant differences between the left and right visual ROIs based on the target locations. Essentially, this GLM analysis tests whether there is an interaction between the direction of search (leftward vs. rightward) and hemisphere (coupling with left visual ROIs vs. coupling with right visual ROIs). This was repeated over all frequencies and time points and done separately for each search type. For ease of interpretation, the outputs of the GLM analysis were represented as $t$ values. In short, this analysis enables us to test whether the frontoparietal network that we had identified selectively couples with early visual areas in a spatially specific way, according to the target location, and whether that happens both when we search a visual display and when we search a remembered display. We used 
a cluster-based permutation testing approach to control for multiple comparisons. We identified any clusters where $t>2.3$ (which approximates an uncorrected threshold of $p=.05$ ), calculated the size of these, and then used a permutation testing approach to test whether any of these clusters were significant. For each permutation, the first steps of the analysis were identical, but at the final GLM stage, the allocation of target location and hemisphere were randomized. We then identified the largest cluster, using the same threshold as in the original analysis. This process was repeated 1000 times, building up a null distribution of cluster sizes that would be expected by chance. Against this distribution, we could then compare the actual effects and derive $p$ values.

\section{Behavioral Analyses}

Behavioral analyses were conducted for each fMRI and MEG experiments separately. First, we analyzed the sensitivity scores $\left[d^{\prime}=Z\right.$ (hit rate) $-Z$ (false alarm rate) $]$ (Green \& Swets, 1966) by a 2 (Search type: visual search, WM search) $\times 2$ (Search load: two-item, four-item) repeated-measures ANOVA. Hit rate was defined as the conditional probability that the participants responded "target present" when the target item was present. False alarm rate was defined as the conditional probability that the participants responded "target-present" when the target item was absent. RTs were also analyzed by a 2 (Search type: visual search, WM search) $\times 2$ (Search load: two-item, four-item) repeated-measures ANOVA. Only correct responses were included for RT analyses.

\section{RESULTS}

\section{Behavioral Results}

Table 1 shows the mean $d^{\prime}$ scores and RTs in each condition for both fMRI and MEG experiments. The $d^{\prime}$ scores showed a significant effect of Search type (fMRI: $F(1,15)=54.23$, $p<.001$; MEG: $F(1,10)=113.04, p<.001)$ and a significant effect of Search load (fMRI: $F(1,15)=39.82, p<.001$;
MEG: $F(1,10)=105.63, p<.001)$. We also observed a significant interaction between Search type and Search load (fMRI: $F(1,15)=13.98, p=.002$; MEG: $F(1,10)=$ $26.51, p<.001)$, indicating a larger difference in $d^{\prime}$ scores between the two-item condition and the four-item condition for WM search (fMRI: $F(1,30)=49.11, p<.001$; MEG: $F(1,20)=118.62, p<.001)$ in contrast to that for visual search (fMRI: $F(1,30)=2.15, p=.15$; MEG: $F(1,20)=$ $12.80, p=.002)$.

The RT data for both experiments showed a significant main effect of Search load (fMRI: $F(1,15)=15.31, p=$ .001 ; MEG: $F(1,10)=71.70, p<.001)$, with faster RTs for two-item than four-item searches. A significant interaction between Search type and Search load was also observed in the MEG experiment, $F(1,10)=6.34, p=.03$. This interaction resulted from a larger RT difference between the two-item and four-item conditions for WM search compared with visual search. No other significant effect or interaction was observed in the fMRI experiment $(p s>.1)$.

\section{fMRI Results}

\section{Common Mechanisms of Target Selection in WM and Perception}

Using a conjunction analysis, we found common activations of the dorsal frontoparietal network for both visual and WM search. These activations included superior, middle, and inferior frontal gyri, FEF, insula/ventrolateral pFC, ACC, and precentral gyrus within the frontal cortex. We also found significant activation in the superior and inferior parietal lobule, intraparietal sulcus, angular gyrus, and precuneus within the posterior parietal cortex. In addition, activations were observed in occipito-temporal areas, including superior, middle, and inferior temporal gyrus, fusiform gyrus, cuneus, and inferior and middle occipital gyrus. Significant subcortical activations also occurred in the thalamus. These results are illustrated in Figure 2 and Table 2. These common activations for both visual and WM search are consistent with previous

Table 1. Mean Sensitivity Scores $\left(d^{\prime}\right)$ and RTs (msec) for fMRI and MEG Experiments Separately

\begin{tabular}{|c|c|c|c|c|}
\hline & \multicolumn{2}{|c|}{ Visual Search } & \multicolumn{2}{|c|}{ WM Search } \\
\hline & Two Items & Four Items & Two Items & Four Items \\
\hline \multicolumn{5}{|c|}{$f M R I$} \\
\hline$d^{\prime}$ & $2.63(0.70)$ & $2.38(0.77)$ & $2.18(0.88)$ & $1.02(0.51)$ \\
\hline RT & $536.67(74.17)$ & $596.28(78.48)$ & $567.47(116.58)$ & 618.96 (106.79) \\
\hline \multicolumn{5}{|c|}{$M E G$} \\
\hline$d^{\prime}$ & $2.49(0.76)$ & $2.15(0.81)$ & $1.95(0.62)$ & $0.90(0.49)$ \\
\hline RT & $407.51(82.17)$ & $482.43(76.43)$ & $417.29(87.46)$ & $516.24(92.94)$ \\
\hline
\end{tabular}

Standard deviations are given in parentheses. 
Table 2. Brain Areas and MNI Coordinates $(x, y, z)$ for Common Activations of Target Selection in Visual and WM Search

\begin{tabular}{lrrrrr}
\hline Brain Areas & & $x$ & $y$ & $z$ & $t$ \\
\hline Frontal Regions & & & & & \\
ACC & $\mathrm{L}$ & -6 & 16 & 40 & 9.45 \\
& $\mathrm{R}$ & 6 & 20 & 44 & 11.06 \\
Superior frontal gyrus & $\mathrm{L}$ & -4 & 10 & 54 & 12.77 \\
& $\mathrm{R}$ & 4 & 10 & 56 & 11.34 \\
Middle frontal gyrus & $\mathrm{L}$ & -42 & 30 & 20 & 5.86 \\
& $\mathrm{R}$ & 42 & 30 & 22 & 7.04 \\
Inferior frontal gyrus & $\mathrm{L}$ & -54 & 8 & 24 & 9.28 \\
& $\mathrm{R}$ & 56 & 8 & 22 & 5.11 \\
FEF & $\mathrm{L}$ & -28 & -6 & 60 & 9.22 \\
& $\mathrm{R}$ & 30 & 0 & 58 & 7.91 \\
Insula/ventrolateral pFC & $\mathrm{L}$ & -34 & 16 & 4 & 11.50 \\
& $\mathrm{R}$ & 33 & 17 & 6 & 10.63 \\
Precentral gyrus & $\mathrm{L}$ & -50 & 4 & 38 & 9.41
\end{tabular}

Parietal Regions

$\begin{array}{lrrrrr}\text { Superior parietal lobule } & \mathrm{L} & -20 & -68 & 56 & 5.66 \\ & \mathrm{R} & 33 & -51 & 48 & 8.59 \\ \text { Inferior parietal lobule } & \mathrm{L} & -46 & -39 & 49 & 8.87 \\ & \mathrm{R} & 38 & -52 & 58 & 9.74 \\ \text { Angular gyrus } & \mathrm{R} & 32 & -60 & 38 & 8.14 \\ \text { Precuneus } & \mathrm{L} & -12 & -72 & 42 & 7.34 \\ & \mathrm{R} & 16 & -70 & 38 & 6.56\end{array}$

Occipito-temporal Regions

\begin{tabular}{lrrrrr} 
Middle occipital gyrus & $\mathrm{L}$ & -36 & -84 & -4 & 3.14 \\
& $\mathrm{R}$ & 34 & -82 & 2 & 2.83 \\
Inferior occipital gyrus & $\mathrm{R}$ & 28 & -82 & -12 & 3.30 \\
Superior temporal gyrus & $\mathrm{L}$ & -50 & -52 & 8 & 3.84 \\
& $\mathrm{R}$ & 40 & -60 & 14 & 2.84 \\
Middle temporal gyrus & $\mathrm{L}$ & -46 & -60 & -8 & 7.91 \\
& $\mathrm{R}$ & 50 & -58 & -8 & 6.22 \\
Inferior temporal gyrus & $\mathrm{R}$ & 48 & -66 & -4 & 6.86 \\
Fusiform gyrus & $\mathrm{L}$ & -36 & -78 & -16 & 3.01 \\
& $\mathrm{R}$ & 48 & -46 & -12 & 5.89 \\
Cuneus & $\mathrm{L}$ & -14 & -70 & 12 & 6.61 \\
& $\mathrm{R}$ & 24 & -72 & 16 & 3.18 \\
Others & & & & & \\
Thalamus & $\mathrm{L}$ & -14 & -20 & 10 & 8.55 \\
& & 8 & -16 & 4 & 7.34 \\
\hline
\end{tabular}

$\mathrm{L}=$ left hemisphere; $\mathrm{R}=$ right hemisphere. findings characterizing the neural mechanisms of attention control in visual search (Anderson, Mannan, Rees, Sumner, \& Kennard, 2010; Egner et al., 2008; Nobre et al., 2003, 2004; Donner et al., 2002).

\section{Functional Visual ROIs}

Analysis of the visual localizer task yielded nonoverlapping visual ROIs in the left and right visual areas in the inferior and middle occipital cortices (see Figure 2). We exploited these lateralized ROIs to guide a subsequent MEG analysis and test for the presence of lateralized modulation of sensory population codes via phase-phase coupling with the frontoparietal network.

\section{MEG Results}

N2pc-like ERF Effect

Analysis of the ERFs revealed the presence of an N2pc-like effect at posterior parietal-occipital sensors, with a relative negativity contralateral to the spatial location of the target. This effect was apparent both in the visual and WM search (Figure $3 \mathrm{~A}$ and $\mathrm{B}$ ). Cluster-based permutation tests revealed significant clusters in both the visual $\left(p_{\text {corrected }}<\right.$ $.001)$ and WM search conditions $\left(p_{\text {corrected }}=.004\right)$.

In a follow-up analysis, we explored whether this effect was modulated by array size. Our design included both two-item and four-item arrays. The initial analysis collapsed across this factor (see also Kuo et al., 2009). Having identified the sensors and time window for the contralateralized ERF effect, we used these to test whether the N2pc effect is modulated by array size. A repeatedmeasures 2 (Target side: contralateral vs. ipsilateral to the target location) $\times 2$ (Search load: two-item, four-item) ANOVA revealed that there was no interaction in the visual search condition, $F(1,10)=0.12, p=.73$. However, there was a significant interaction in the WM search condition, $F(1,10)=5.59, p=.04$, with the N2pc-like effect being smaller on the four-item trials, relative to two-item trials. These data can be seen in Figure 3C.

\section{Spatially Specific Phase-Phase Coupling}

The phase-coupling analysis examining the functional connectivity in ongoing oscillatory activity between the frontoparietal network and early visual areas for both search types (these areas can be seen in Figure 4A) revealed a spatially specific modulation of phase coupling for both search types at around 200 msec. When participants searched a visual display for the target, we observed a relative increase in coupling with the visual area contralateral to the location of that target. This was apparent in three significant cluster effects (all $p_{\text {corrected }}<.001$ ), centered on $5.5 \mathrm{~Hz}$ (at $242 \mathrm{msec}$ ), $11 \mathrm{~Hz}$ (at $344 \mathrm{msec}$ ), and $12.5 \mathrm{~Hz}$ (at $228 \mathrm{msec}$ ). These effects can be seen in Figure 4B. The mean coupling values taken at the peak 


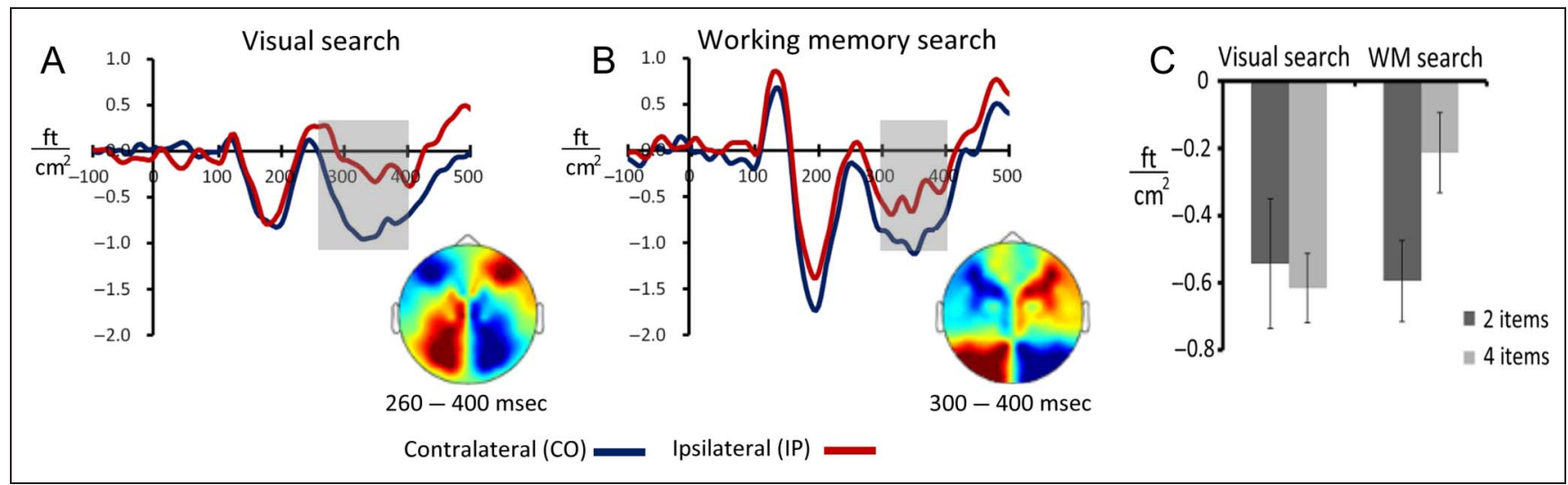

Figure 3. Sensor space ERF results of MEG experiment. The N2pc-like ERF effects in the (A) visual search and (B) WM search showed similar time courses (grand-averaged waveforms) and topographies at the posterior latitudinal gradiometers contralateral (CO, blue lines) and ipsilateral (IP, red lines) to the side of the target. The topographic maps exhibited the lateralized differences in magnetic field between contralateral (blue/right side) and ipsilateral sites (red/left side). These maps show the symmetrical relative differences in magnetic field, which is more negative over the contralateral scalp and more positive over the ipsilateral scalp. (C) In a follow-up analysis, we showed that this N2pc-like ERF effect was modulated by array size for WM search but not for visual search, indicating a smaller N2pc on the four-item trials compared with two-item trials.

of this effect $(12.5 \mathrm{~Hz}$ at $228 \mathrm{msec})$ can be seen in Figure 4C. When participants searched a remembered display for the target, we observed a similar relative increase in coupling with the contralateral visual area, relative to the target location. This was apparent as three significant clusters (all $\left.p_{\text {corrected }}<.001\right)$. These were centered on $7.5 \mathrm{~Hz}$ (at $204 \mathrm{msec}$ ), $13.5 \mathrm{~Hz}$ (at $214 \mathrm{msec}$ ), and $17.5 \mathrm{~Hz}$ (at $210 \mathrm{msec}$ ). These clusters can be seen in Figure 4E. The mean coupling values taken at the peak of this effect (freq $=7.5 \mathrm{~Hz}$, time $=204 \mathrm{msec}$ ) can be seen in Figure 4F. In summary, when participants searched for a target, either within a visual or remembered display, we observed a change in the strength of phase coupling, with it becoming relatively stronger contralateral the target location.

In a follow-up analysis, we explored whether the strength of this contralateralized coupling was modulated by array size. To do this, we repeated the analysis described already, but separately for two- versus four-item trials. In the GLM, in addition to a regressor that captured the degree of contralateralization, we included a regressor that corresponded to array size. This enabled us to test the strength of the interaction between these two regressors. We used the same thresholding and permutation testing approach described already for testing for significance. In the visual search condition, the contralateralized coupling effect was not significantly modulated by array size. This can be seen Figure 4D. By contrast, the contralateralized coupling effect seen in the WM search condition was significantly modulated by array size, being stronger when participants were holding four items in mind, relative to when they were holding two items in mind (Figure $4 \mathrm{G}$ ). There were four significant clusters, all in proximity to one another: The first was centered at $154 \mathrm{msec}$ and $15.25 \mathrm{~Hz}$, the second at $193 \mathrm{msec}$ and $17.25 \mathrm{~Hz}$, the third at $201 \mathrm{msec}$ and $16.5 \mathrm{~Hz}$, and the last at $223 \mathrm{msec}$ and $16.25 \mathrm{~Hz}$.

\section{Phase-coupling and ERF Correlation Analysis}

A final trial-to-trial analysis explored the potential overlap between our two MEG findings. We tested whether the relative contralaterality of the source space phase-phase coupling was significantly associated with the N2pc-like sensor space effect. To do this, we calculated a single trial measure of phase-phase coupling in the same way as before, but without collapsing across trials. We also calculated the single trial analog of the N2pc-like sensor space effect, as the relative contralaterality of the magnetic field between 200 and 400 msec (contralateral minus ipsilateral). We used the sensors that were established by our cluster-based approach in the sensor space result for each search type. We then performed a trial-wise GLM, with the relative contralaterality in phase-phase coupling being the outcome measure and the sensor space N2pclike effect as the predictor. This was repeated over all combinations of frequency and time for each individual participant. We restricted ourselves to the $200-400 \mathrm{msec}$ time window and $8-20 \mathrm{~Hz}$ frequency range when doing this. This wider frequency range was used because in previous studies we have observed functional coupling in the alpha range (Barnes, Woolrich, Baker, Colclough, \& Astle, 2016) and in the lower beta range (Astle, Barnes, Baker, Colclough, \& Woolrich, 2015; Astle, Luckhoo, et al., 2015), and other researchers have specifically highlighted individual differences in peak alpha frequency (Klimesch, Sauseng, \& Gerloff, 2003; Osaka, Osaka, Koyama, Okusa, \& Kakigi, 1999). We then pooled the results across our participants in a second level analysis, which was equivalent to a one-sample $t$ test. The results of this can be seen in Figure 5A for the visual search condition and Figure 5B for the WM search condition. In both cases, there was a large negative cluster, indicating that the degree of contralateralization in the phase-phase coupling is significantly 
associated with relative contralateral negativity in the sensor space result. In short, on trials when phase-phase coupling is particularly biased to one hemisphere, there also tends to be a larger N2pc effect. We used the same clustering threshold as before $(t>2.3)$ and used a sign-flipping permutation approach to test for the significance of these effects. In the visual search condition, the effect was centered on $14.5 \mathrm{~Hz}$ (at $282 \mathrm{msec}$ ); in the WM search condition, the effect was centered on $17 \mathrm{~Hz}$ (at $250 \mathrm{msec}$ ). However, only the WM search condition survived the permutation correction $\left[p_{\text {corrected }}=.0290\right]$, with the visual search condition being nonsignificant $\left[p_{\text {corrected }}=.1100\right]$.

\section{DISCUSSION}

The purpose of this study was to establish when and how frontoparietal networks are involved in visual and WM search. We exploited the contralateral organization of the human visual system to explore the neurophysiological mechanisms underpinning spatial biasing during both search types. Our data show that, during visual and mnemonic search, lateral frontoparietal networks are coordinated with early visual areas, in a temporally and spatially specific fashion, depending upon the location of the searched-for target. This spatiotopic coordination is apparent in phase-phase coupling, and this effect precedes a sensor level marker of target selection-the N2pc.

The behavioral data from both of our experiments show a highly consistent pattern of performance-searching WM is more difficult and is more affected by set size than its visual counterpart. This pattern replicates previous studies (e.g., Kuo et al., 2009). We also replicate previous demonstrations of an N2pc during WM search (Eimer, 2014; Dell'Acqua et al., 2010; Eimer \& Kiss, 2010; Astle et al., 2009; Kuo et al., 2009), adding to a growing body of research demonstrating that searching internal representations recruits spatially specific mechanisms comparable to those used in visual search. In the current data set, the pattern of the N2pc effect mirrored the overall

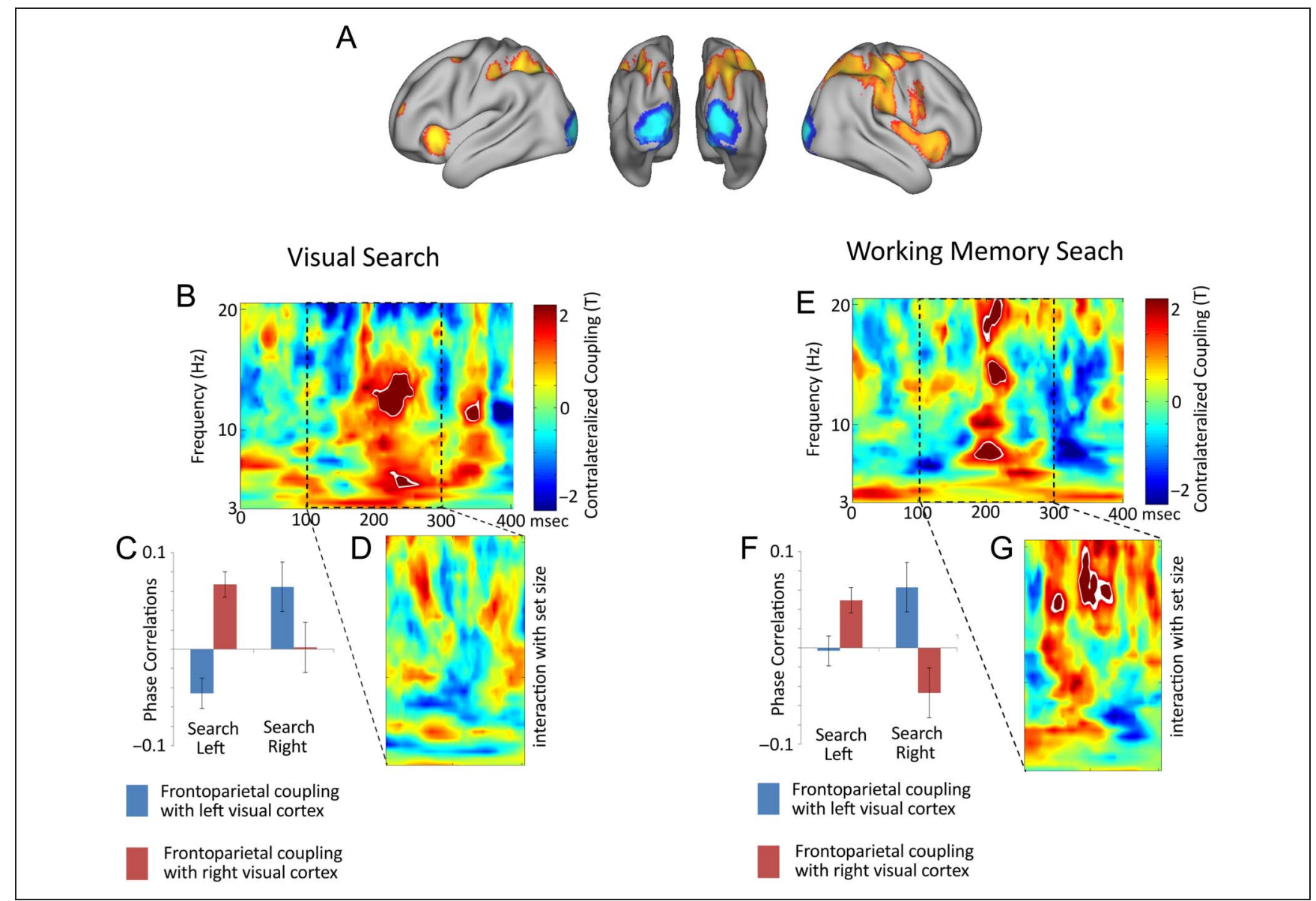

Figure 4. Spatially specific phase-phase coupling results. (A) Our phase-coupling analysis tested the functional connectivity in ongoing oscillatory activity between the frontoparietal network and early visual areas for both visual and WM search and revealed a spatially specific modulation of phase coupling for both search types at around $200 \mathrm{msec}$. (B) On visual search trials, we observed a relative increase in coupling with the visual area contralateral to the location of the target, centered on $5.5 \mathrm{~Hz}$ (at $242 \mathrm{msec}$ ), $11 \mathrm{~Hz}$ (at $344 \mathrm{msec}$ ), and $12.5 \mathrm{~Hz}$ (at $228 \mathrm{msec}$ ). (C) The mean coupling values taken at the peak of the effects for visual search. (D) The contralteralized coupling effect was not modulated by array size for visual search. (E) On WM search trials, we observed a similar relative increase in coupling with the contralateral visual area relative to the target location, centered on $7.5 \mathrm{~Hz}$ (at $204 \mathrm{msec}$ ), $13.5 \mathrm{~Hz}$ (at $214 \mathrm{msec}$ ) and $17.5 \mathrm{~Hz}$ (at $210 \mathrm{msec}$ ). (F) The mean coupling values taken at the peak of the effects for WM search. (G) The contralteralized coupling effect was significantly modulated by array size for WM search. 


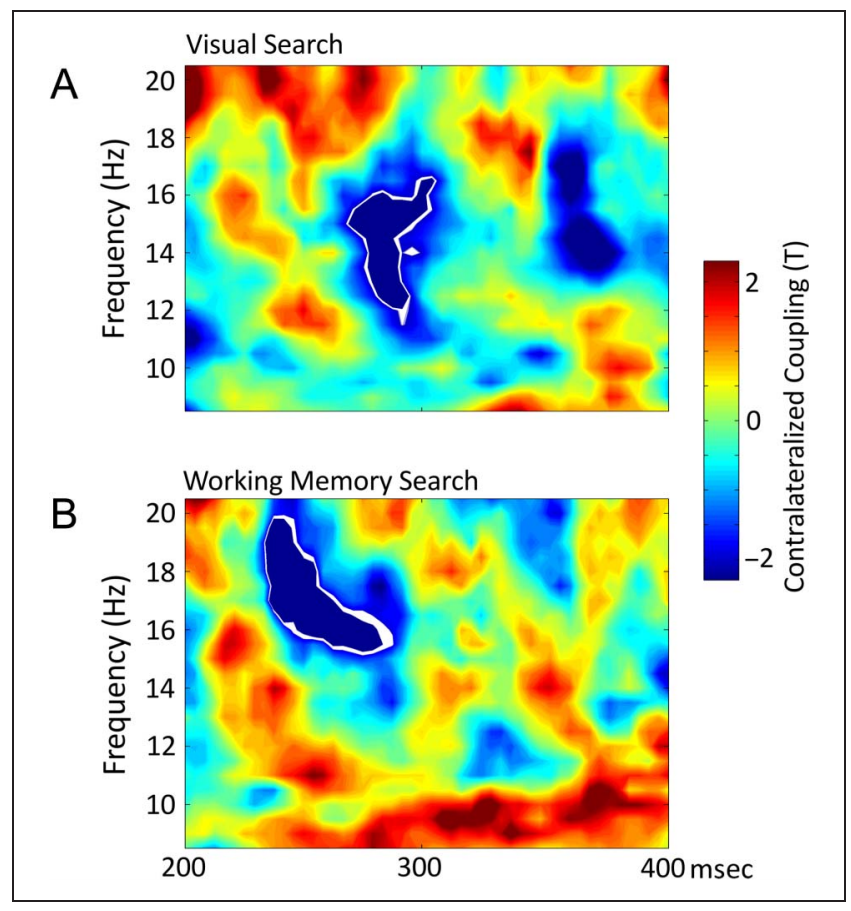

Figure 5. Phase-coupling and ERF correlation results. We showed that the relative contralaterality of the source space phase-phase coupling was significantly correlated with the N2pc-like sensor space effects on a trial-by-trial basis for visual search (A) and WM search (B). In the visual search condition, the effect was centered on $14.5 \mathrm{~Hz}$ (at $282 \mathrm{msec}$ ); in the WM search condition, the effect was centered on $17 \mathrm{~Hz}$ (at $250 \mathrm{msec}$ ). However, only the WM search condition survived the permutation correction for the contralateralized coupling.

pattern of the behavioral data: There was a strong effect of array size in the WM search condition, relative to the visual search condition. This pattern and that of the behavioral data may reflect the limited capacity of WM relative to sensory processing. This additional constraint may make the search process more difficult or more protracted when WM is more heavily loaded. We further extend these findings by showing that both search types recruit a common network of frontoparietal areas, including the superior, middle, and inferior frontal gyri, FEF, insula/ventrolateral pFC, ACC, superior and inferior parietal lobule, intraparietal sulcus, and precuneus. Although this has not been shown previously, it is consistent with a number of findings that emphasize the overlapping neural systems of memory and attentional selection (Fusser et al., 2011; Nee \& Jonides, 2009; Roth, Johnson, Raye, \& Constable, 2009; Mayer et al., 2007; Awh \& Jonides, 2001). For example, a recent fMRI study showed common activation in a distributed frontal, parietal, and visual areas for attention-demanding visual search and WM encoding (Mayer et al., 2007). Taken together, the combination of our sensor level MEG effects and the large number of common brains areas established by the fMRI analysis underscores the involvement of a comparable attentional control process when selecting targets from external and internal representations. The contralateralized organiza- tion of the N2pc effect, reflecting the organization of the original perceptual material in both conditions, suggests that the process of selection in both perceptual and memory search involves the activation of spatiotopic sensory codes. This spatiotopic selection process within WM is more difficult when load increases, reflected in both the behavioral data and the pattern of N2pc effects for the WM condition specifically. We tested whether and how this activation of spatiotopic sensory codes might occur in our subsequent fMRI-guided MEG analysis.

Combining the anatomically precise information from fMRI, including visual localizer scans, with the dynamic electrophysiological activity captured by MEG, we identified how and when frontoparietal cortex is coordinated with sensory processing areas during the search process. Phase-phase coupling provides a mechanism by which this coordination occurs, with the frontoparietal network coupling selectively with the early visual areas contralateral to the target location. The timing of this process is largely consistent across both search types, occurring at around $200 \mathrm{msec}$ and therefore preceding the onset of the sensor level N2pc effect. Like the sensor level N2pc effects, while this coupling occurred in both search types, there were important differences with regard their sensitivity to array size effects. During WM search, the coupling was more strongly contralateralized at the higher load level (four items vs. two items), whereas the effect during visual search was not sensitive to this manipulation. Finally, the source level phase-phase coupling was significantly associated with the sensor level N2pc effect on a trial-by-trial basis; trials that showed a strong spatial basis in the strength of coupling also tended to show a strong N2pc effect. We speculate that these two electrophysiological effects reflect aspects of a spatial biasing process that operates via the activation of sensory codes, beginning with phase-phase coupling and ending with the N2pc effect. Specifically, template-based information contained in control regions within frontoparietal cortex participates in the spatiotopic modulation of communication with the early visual areas via this altered pattern of coupling. In turn, this altered communication facilitates target selection, with the final template-target match being indexed by the N2pc. A greater degree of spatiotopic modulation is required when searching within four WM items, and the subsequent template-target match process is attenuated. In both cases, this may reflect the reduced representational quality of those items (Murray, Nobre, Clark, Cravo, \& Stokes, 2013) - it is more difficult to activate the spatiotopic sensory codes via phase-coupling, and the final selection process is weaker when at the limits of WM capacity. With the current data alone, we cannot rule out the possible role of some earlier process that highlights, perhaps preattentively, the candidate target location: The mechanisms we describe could be responsible for the enhancement and selection of the target once a candidate location is established; alternatively, spatiotopic modulation of communication with 
visual areas could itself reflect the emerging localization of the target item.

Recent studies using functional or effective connectivity analyses have shown that the prefrontal or parietal regions can selectively modulate processing of task-relevant perceptual inputs and WM representations by biasing neural activity in early visual cortex (Kuo et al., 2014; Zanto, Rubens, Thangavel, \& Gazzaley, 2011; Bressler, Tang, Sylvester, Shulman, \& Corbetta, 2008). Using MEG, we show that this functional coupling is highly dynamic and can be modulated in a temporally and spatially specific fashion to accommodate the changing location of the target. This account is functionally similar to the notion of communication through coherence (Fries, 2005, 2015). In this framework phase, relationships between cortical areas provide a means by which the information flow through the processing stream can be gated. By aligning the phase of successive processing centers, relevant information can be allowed to pass along the processing hierarchy, whereas representations held in sensory areas oscillating asynchronously are lost. This cross-modality approach enables us to link the mechanisms revealed by nonhuman primate intracranial recordings sensor level effects and source space electromagnetic measures in humans. We also extend recent findings from nonhuman primates that implicate the role of synchronization in lowfrequency oscillatory activity in enhancing interregional communication for both selective attention and WM (Fries, 2015; Womelsdorf \& Fries, 2007; Womelsdorf et al., 2007). An important next step in applying these analytical approaches to MEG data is to capitalize on the technique's high temporal resolution. With the current pipeline, we cannot establish which areas (if any) take a lead role in this interregional communication, and in future, the temporal information will be necessary in establishing the order of any coupling changes.

These rhythms may be able to play this coordinating role because they reflect the excitatory and inhibitory firing patterns of local neuronal populations (Klimesch, Sauseng, \& Hanslmayr, 2007). In particular, the alpha rhythm has been shown to modulate according to the relative priority and behavioral relevance of a target (Bonnefond \& Jensen, 2012; Jensen, Bonnefond, \& VanRullen, 2012). That said, this role is not unique to alpha, with a number of studies showing that the relative coordination of slow frequency oscillatory rhythms in the theta (Astle, Luckhoo, et al., 2015), alpha (Zumer, Scheeringa, Schoffelen, Norris, \& Jensen, 2014; Bonnefond \& Jensen, 2012), and lower beta rhythm (Astle, Barnes, et al., 2015) are strong predictors of the efficiency of attentional and WM processes. However, caution is needed when applying this interpretation to our findings: our phase-phase coupling effects are seen at different frequencies within the theta-alpha range, and with these data alone, we cannot tell whether this punctate change in phase coupling reflects changes in the underlying electrophysiological rhythms or a change in the phase relation between nonrhythmic neural signals in frontopa- rietal cortex and visual areas, which is reflected within the theta-alpha range when analyzed using a time-frequency decomposition.

In summary, the purpose of this study was to explore the common mechanisms and principles involved in perceptual and mnemonic search. It is unlikely that these processes are identical, with capacity limits providing a differential constraint on WM search. However, we show that both types of search involve the activation of early sensory codes, in a spatially and temporally specific fashion, and that phase-phase coupling provides a mechanism by which this activation occurs.

\section{Acknowledgments}

The research supported by the National Institute for Health Research Oxford Biomedical Research Centre based at Oxford University Hospitals Trust Oxford University, an MRC UK MEG Partnership grant MR/K005464/1, and a Wellcome Trust Senior Investigator Award (A. C. N.) 104571/Z/14/Z. G. S. was supported by the John Fell Oxford University Press Fund and the James S. McDonnell Foundation Understanding Human Cognition Scholar Award. B. C. K. was supported by a grant from the Ministry of Science and Technology, Taiwan (NSC 103-2420-H002-010-MY2). D. E. A. was supported by the Medical Research Council (United Kingdom) intramural program (MC-A0605PQ40)

Reprint requests should be sent to Duncan E. Astle, MRC Cognition and Brain Sciences Unit, 15 Chaucer Road, Cambridge, CB2 7EF, United Kingdom, or via e-mail: duncan.astle@mrccbu.cam.ac.uk.

\section{REFERENCES}

Anderson, E. J., Mannan, S. K., Rees, G., Sumner, P., \& Kennard, C. (2010). Overlapping functional anatomy for working memory and visual search. Experimental Brain Research, 200, 91-107.

Astle, D. E., Barnes, J. J., Baker, K., Colclough, G. L., \& Woolrich, M. W. (2015). Cognitive training enhances intrinsic brain connectivity in childhood. Journal of Neuroscience, 35, 6277-6283.

Astle, D. E., Luckhoo, H., Woolrich, M., Kuo, B.-C., Nobre, A. C., \& Scerif, G. (2015). The neural dynamics of frontoparietal networks in childhood revealed using magnetoencephalography. Cerebral Cortex, 25, 3868-3876.

Astle, D. E., Scerif, G., Kuo, B.-C., \& Nobre, A. C. (2009). Spatial selection of features within perceived and remembered objects. Frontiers in Human Neuroscience, 3, 1-9.

Awh, E., \& Jonides, J. (2001). Overlapping mechanisms of attention and spatial working memory. Trends in Cognitive Sciences, 5, 119-126.

Barnes, J. J., Woolrich, M. W., Baker, K., Colclough, G. L., \& Astle, D. E. (2016). Electrophysiological measures of resting state functional connectivity and their relationship with working memory capacity in childhood. Developmental Science, 19, 19-31.

Becker, S. I., Grubert, A., \& Dux, P. E. (2014). Distinct neural networks for target feature versus dimension changes in visual search, as revealed by EEG and fMRI. Neuroimage, 102, 798-808.

Bonnefond, M., \& Jensen, O. (2012). Alpha oscillations serve to protect working memory maintenance against anticipated distracters. Current Biology, 22, 1969-1974. 
Bressler, S. L., Tang, W., Sylvester, C. M., Shulman, G. L., \& Corbetta, M. (2008). Top-down control of human visual cortex by frontal and parietal cortex in anticipatory visual spatial attention. Journal of Neuroscience, 28, 10056-10061.

Brignani, D., Lepsien, J., \& Nobre, A. C. (2010). Purely endogenous capture of attention by task-defining features proceeds independently from spatial attention. Neuroimage, 51, 859-866.

Buschman, T. J., \& Miller, E. K. (2007). Top-down versus bottom-up control of attention in the prefrontal and posterior parietal cortices. Science, 315, 1860-1862.

Chelazzi, L. (1999). Serial attention mechanisms in visual search: A critical look at the evidence. Psychological Research, 62, 195-219.

Chelazzi, L., Duncan, J., Miller, E. K., \& Desimone, R. (1998). Responses of neurons in inferior temporal cortex during memory-guided visual search. Journal of Neurophysiology, 80, 2918-2940.

Chelazzi, L., Miller, E. K., Duncan, J., \& Desimone, R. (1993). A neural basis for visual search in inferior temporal cortex. Nature, 363, 345-347.

Collins, D. L., Neelin, P., Peters, T. M., \& Evans, A. C. (1994). Automatic 3D intersubject registration of MR volumetric data in standardized Talairach space. Journal of Computer Assisted Tomography, 18, 192-205.

Corbetta, M., Shulman, G. L., Miezin, F. M., \& Petersen, S. (1995). Superior parietal cortex activation during spatial attention shifts and visual feature conjunction. Science, 270, 802-805.

Cunningham, C. A., \& Wolfe, J. M. (2014). The role of object categories in hybrid visual and memory search. Journal of Experimental Psychology: General, 143, 1585-1599.

Dell'Acqua, R., Sessa, P., Toffanin, P., Luria, R., \& Jolicoeur, P (2010). Orienting attention to objects in visual short-term memory. Neuropsychologia, 48, 419-428.

Desimone, R., \& Duncan, J. (1995). Neural mechanisms of selective visual attention. Annual Review of Neuroscience, 18, 193-222.

Donner, T. H., Kettermann, A., Diesch, E., Ostendorf, F., Villringer, A., \& Brandt, S. A. (2002). Visual feature and conjunction searches of equal difficulty engage only partially overlapping frontoparietal networks. Neuroimage, $15,16-25$

Duncan, J., \& Humphreys, G. W. (1989). Visual search and stimulus similarity. Psychological Review, 96, 433-458.

Egner, T., Monti, J. M. P., Trittschuh, E. H., Wieneke, C. A., Hirsch, J., \& Mesulam, M.-M. (2008). Neural integration of top-down spatial and feature-based information in visual search. Journal of Neuroscience, 28, 6141-6151.

Eimer, M. (1996). The N2pc component as an indicator of attentional selectivity. Electroencephalography and Clinical Neurophysiology, 99, 225-234.

Eimer, M. (2014). The neural basis of attentional control in visual search. Trends in Cognitive Sciences, 18, 526-535.

Eimer, M., \& Grubert, A. (2014). Spatial attention can be allocated rapidly and in parallel to new visual objects. Current Biology, 24, 193-198.

Eimer, M., \& Kiss, M. (2010). An electrophysiological measure of access to representations in visual working memory. Psychophysiology, 47, 197-200.

Endo, N., Saiki, J., Nakao, Y., \& Saito, H. (2003). Perceptual judgments of novel contour shapes and hierarchical descriptions of geometrical properties. The Japanese Journal of Psychology, 74, 346-353.

Fries, P. (2005). A mechanism for cognitive dynamics: Neuronal communication through neuronal coherence. Trends in Cognitive Sciences, 9, 474-480.
Fries, P. (2009). Neuronal gamma-band synchronization as a fundamental process in cortical computation. Annual Review of Neuroscience, 32, 209-224.

Fries, P. (2015). Rhythms for cognition: Communication through coherence. Neuron, 88, 220-235.

Fries, P., Reynolds, J. H., Rorie, A. E., \& Desimone, R. (2001). Modulation of oscillatory neuronal synchronization by selective visual attention. Science, 291, 1560-1563.

Fusser, F., Linden, D. E. J., Rahm, B., Hampel, H., Haenschel, C., \& Mayer, J. S. (2011). Common capacity-limited neural mechanisms of selective attention and spatial working memory encoding. European Journal of Neuroscience, 34, 827-838.

Green, D. M., \& Swets, J. A. (1966). Signal detection theory and psychophysics. Huntington, NY: Robert E. Krieger Publishing Company.

Hopfinger, J. B., Buchel, C., Holmes, A. P., \& Friston, K. J. (2000). A study of analysis parameters that influence the sensitivity of event-related fMRI analyses. Neuroimage, 11, 326-333.

Jensen, O., Bonnefond, M., \& VanRullen, R. (2012). An oscillatory mechanism for prioritizing salient unattended stimuli. Trends in Cognitive Sciences, 16, 200-206.

Kanwisher, N., \& Wojciulik, E. (2000). Visual attention: Insights from brain imaging. Nature Reviews Neuroscience, 1, 91-100.

Klimesch, W. (2012). Alpha-band oscillations, attention, and controlled access to stored information. Trends in Cognitive Sciences, 16, 606-617.

Klimesch, W., Sauseng, P., \& Gerloff, C. (2003). Enhancing cognitive performance with repetitive transcranial magnetic stimulation at human individual alpha frequency. European Journal of Neuroscience, 17, 1129-1133.

Klimesch, W., Sauseng, P., \& Hanslmayr, S. (2007). EEG alpha oscillations: The inhibition-timing hypothesis. Brain Research Reviews, 53, 63-88.

Kuo, B.-C., Rao, A., Lepsien, J., \& Nobre, A. C. (2009). Searching for targets within the spatial layout of visual short-term memory. Journal of Neuroscience, 29, 8032-8038.

Kuo, B.-C., Stokes, M. G., Murray, A. M., \& Nobre, A. C. (2014). Attention biases visual activity in visual short-term memory. Journal of Cognitive Neuroscience, 26, 1377-1389.

Luck, S. J., \& Hillyard, S. A. (1994). Spatial filtering during visual search: Evidence from human electrophysiology. Journal of Experimental Psychology: Human Perception and Performance, 20, 1000-1014.

Luckhoo, H., Hale, J. R., Stokes, M. G., Nobre, A. C., Morris, P. G., Brookes, M. J., et al. (2012). Inferring task-related networks using independent component analysis in magnetoencephalography. Neuroimage, 62, 530-541.

Maris, E., \& Oostenveld, R. (2007). Nonparametric statistical testing of EEG- and MEG-data. Journal of Neuroscience Methods, 164, 177-190.

Marshall, T. R., Bergmann, T. O., \& Jensen, O. (2015). Frontoparietal structural connectivity mediates the top-down control of neuronal synchronization associated with selective attention. PLoS Biology, 13, e1002272.

Mayer, J. S., Bittner, R. A., Nikolić, D., Bledowski, C., Goebel, R., \& Linden, D. E. J. (2007). Common neural substrates for visual working memory and attention. Neuroimage, 36, 441-453.

Miller, E. K., \& Cohen, J. D. (2001). An integrative theory of prefrontal cortex function. Annual Review of Neuroscience, 24, 167-202.

Mitchell, D. J., \& Cusack, R. (2011). The temporal evolution of electromagnetic markers sensitive to the capacity limits of visual short-term memory. Frontiers in Human Neuroscience, 5, 18. 
Murray, A. M., Nobre, A. C., Clark, I. A., Cravo, A. M., \& Stokes, M. G. (2013). Attention restores discrete items to visual short-term memory. Psychological Science, 24, 550-556.

Nee, D. E., \& Jonides, J. (2009). Common and distinct neural correlates of perceptual and memorial selection. Neuroimage, 45, 963-975.

Nichols, T., Brett, M., Andersson, J., Wager, T., \& Poline, J.-B. (2005). Valid conjunction inference with the minimum statistic. Neuroimage, 25, 653-660.

Nobre, A. C., Coull, J. T., Maquet, P., Frith, C. D., Vandenberghe, R., \& Mesulam, M. M. (2004). Orienting attention to locations in perceptual versus mental representations. Journal of Cognitive Neuroscience, 16, 363-373.

Nobre, A. C., Coull, J. T., Walsh, V., \& Frith, C. D. (2003). Brain activations during visual search: Contributions of search efficiency versus feature binding. Neuroimage, 18, 91-103.

Oldfield, R. C. (1971). The assessment and analysis of handedness: The Edinburgh inventory. Neuropsychologia, 9, 97-113.

Oostenveld, R., Fries, P., Maris, E., \& Schoffelen, J.-M. (2011). FieldTrip: Open source software for advanced analysis of MEG, EEG, and invasive electrophysiological data. Computational Intelligence and Neuroscience, 2011, 9.

Osaka, M., Osaka, N., Koyama, S., Okusa, T., \& Kakigi, R. (1999). Individual differences in working memory and the peak alpha frequency shift on magnetoencephalography. Cognitive Brain Research, 8, 365-368.

Pauen, K., \& Ivanova, G. (2013). Multiple circular-circular correlation coefficients for the quantification of phase synchronization processes in the brain. Biomedizinische Technik/Biomedical Engineering, 58, 141-155.

Preston, T. J., Guo, F., Das, K., Giesbrecht, B., \& Eckstein, M. P. (2013). Neural representations of contextual guidance in visual search of real-world scenes. Journal of Neuroscience, 33, 7846-7855.

Roth, J. K., Johnson, M. K., Raye, C. L., \& Constable, R. T. (2009). Similar and dissociable mechanisms for attention to internal versus external information. Neuroimage, 48, 601-608.

Siegel, M., Donner, T. H., Oostenveld, R., Fries, P., \& Engel, A. K. (2008). Neuronal synchronization along the dorsal visual pathway reflects the focus of spatial attention. Neuron, 60, 709-719.

Tollner, T., Gramann, K., Muller, H. J., Kiss, M., \& Eimer, M. (2008). Electrophysiological markers of visual dimension changes and response changes. Journal of Experimental
Psychology: Human Perception and Performance, 34, 531-542.

Treisman, A. M., \& Gelade, G. (1980). A feature-integration theory of attention. Cognitive Psychology, 12, 97-136.

Treisman, A. M., \& Sato, S. (1990). Conjunction search revisited. Journal of Experimental Psychology: Human Perception and Performance, 16, 459-478.

Weidner, R., Krummenacher, J., Reimann, B., Muller, H. J., \& Fink, G. R. (2009). Sources of top-down control in visual search. Journal of Cognitive Neuroscience, 21, 2100-2113.

Wolfe, J. M. (1994). Guided Search 2.0 A revised model of visual search. Psychonomic Bulletin \& Review, 1, 202-238.

Wolfe, J. M., Cave, K. R., \& Franzel, S. L. (1989). Guided search: An alternative to the feature integration model for visual search. Journal of Experimental Psychology: Human Perception and Performance, 15, 419-433.

Wolfe, J. M., Võ, M. L. H., Evans, K. K., \& Greene, M. R. (2011). Visual search in scenes involves selective and nonselective pathways. Trends in Cognitive Sciences, 15, 77-84.

Womelsdorf, T., \& Fries, P. (2007). The role of neuronal synchronization in selective attention. Current Opinion in Neurobiology, 17, 154-160.

Womelsdorf, T., Schoffelen, J.-M., Oostenveld, R., Singer, W., Desimone, R., Engel, A. K., et al. (2007). Modulation of neuronal interactions through neuronal synchronization. Science, 316, 1609-1612.

Woodman, G. F., Kang, M.-S., Rossi, A. F., \& Schall, J. D. (2007). Nonhuman primate event-related potentials indexing covert shifts of attention. Proceedings of the National Academy of Sciences, U.S.A., 104, 15111-15116.

Woolrich, M., Hunt, L., Groves, A., \& Barnes, G. (2011). MEG beamforming using Bayesian PCA for adaptive data covariance matrix regularization. Neuroimage, 57, 1466-1479.

Worsley, K. J., Marrett, S., Neelin, P., Vandal, A., Friston, K. J., \& Evans, A. (1996). A unified statistical approach for determining significant signals in images of cerebral activation. Human Brain Mapping, 4, 58-73.

Zanto, T. P., Rubens, M. T., Thangavel, A., \& Gazzaley, A. (2011). Causal role of the prefrontal cortex in top-down modulation of visual processing and working memory. Nature Neuroscience, 14, 656-661.

Zumer, J. M., Scheeringa, R., Schoffelen, J.-M., Norris, D. G., \& Jensen, O. (2014). Occipital alpha activity during stimulus processing gates the information flow to object-selective cortex. PLoS Biology, 12, e1001965. 